Short communication

\title{
Reactivity to AQP4 epitopes in relapsing-remitting multiple sclerosis
}

\author{
H. Alexopoulos a,*, E.I. Kampylafka ${ }^{\text {a }}$, I. Chatzi a,b, M. Travasarou ${ }^{\text {b }}$, K.E. Karageorgiou ${ }^{\text {b }}$, \\ M.C. Dalakas ${ }^{\text {a, } * *,}$, A.G. Tzioufas ${ }^{\text {a }}$ \\ a Neuroimmunology Unit, Department of Pathophysiology, Faculty of Medicine, National and Kapodistrian University of Athens, Greece \\ b Department of Neurology, Athens General Hospital, "G.Genimatas", Greece
}

\section{A R T I C L E I N F O}

\section{Article history:}

Received 18 January 2013

Received in revised form 11 April 2013

Accepted 17 April 2013

\section{Keywords:}

Multiple sclerosis

Epitope mapping

Aquaporin-4

Astrocytes

Demyelination

\begin{abstract}
A B S T R A C T
Autoantibodies against the water channel AQP4, expressed predominately in central nervous system astrocytes, are markers and pathogenic factors in Devic's disease. In this study we examined whether Multiple Sclerosis (MS) patients recognize antigenic epitopes on AQP4 that may define distinct disease subsets. We screened sera from 45 patients with relapsing-remitting MS (RRMS) and 13 patients with primary progressive MS (PMS). 23 Neuromyelitis Optica (NMO) patients previously characterized were used as assay positive/negative controls. Sera from 23 patients with Systemic Lupus Erythematosus, 23 with primary Sjogren syndrome without neurological involvement and from 28 healthy individuals were also used as controls. NMO-positive sera exhibited reactivity against the intracellular epitope AQPaa252-275, confirming previous observations. All RRMS sera tested negative for anti-AQP4 antibodies using a cell-based assay, but surprisingly, $13 \%$ of them reacted with the epitope AQPaa252-275. PMS, healthy and disease controls showed no specific reactivity. Whether these antibodies define distinct MS subsets and have a pathogenic potential pointing to convergent pathogenetic mechanism with NMO, or are simply markers of astrocytic damage, remains to be determined.
\end{abstract}

(C) 2013 Elsevier B.V. All rights reserved.

\section{Introduction}

Multiple sclerosis (MS) is a chronic debilitating autoimmune disease of the central nervous system. It is considered a T-cell orchestrated disorder, although significant evidence supports the notion that B-cell autoimmunity is an important contributor. Histopathological studies have shown the existence of B-cell mediated type II MS (Lucchinetti et al., 2000) and serological studies have described a number of autoantibodies, the most typical being the antibodies against MOG and the most recent being the antibodies against ion channel Kir4.1 (Srivastava et al., 2012). In contrast, in Neuromyelitis optica (NMO) or Devic's disease the main pathogenetic factor is the antibodies against AQP4, a water channel mainly expressed in astrocytes (Lennon et al., 2005). On the basis of distinct pathogenetic mechanisms, MS and NMO are considered different disease entities, despite certain radiological and clinical similarities as well as some recent overlapping data that cloud the disease boundaries (Bruck et al., 2012; Siritho et al., 2011). The suggestion that there may be clinical intermediate types

\footnotetext{
* Correspondence to: H. Alexopoulos, Neuroimmunology Unit, Department of Pathophysiology, Faculty of Medicine, National and Kapodistrian University of Athens, 75 Mikras Asias Street, Athens, 11527, Greece. Tel.: +30 2107462720.

** Correspondence to: M.C. Dalakas, Neuroimmunology Unit, Department of Pathophysiology, Faculty of Medicine, National and Kapodistrian University of Athens, 75 Mikras Asias Street, Athens, 11527, Greece. Tel.: + 302107462616.

E-mail addresses: halexo@med.uoa.gr (H. Alexopoulos), mdalakas@med.uoa.gr (M.C. Dalakas).
}

within the spectrum of NMO and MS subsets, prompted us to investigate whether we could detect signs of NMO autoimmunity in typical MS patients. A sensitive ELISA method, recently developed by our group (Kampylafka et al., 2011) to describe the dominant B-cell linear epitopes in NMO-AQP4 positive patients, was employed.

\section{Methods}

\subsection{Patients and sera}

Sera was tested from 45 patients with relapsing-remitting MS (RRMS) and 13 patients with primary progressive MS (PMS) diagnosed according to McDonald criteria. 23 patients positive for anti-AQP4 antibodies were selected using a commercially available cell based assay (Euroimmun, Luebeck, Germany). Sera from 28 healthy subjects were used as normal controls. The disease control group comprised of 23 patients with Systemic Lupus Erythematosus (SLE) fulfilling the 1997 American College of Rheumatology revised criteria for the classification of SLE (Hochberg, 1997) and 23 patients with primary Sjögren's Syndrome, without neurological involvement fulfilling the AmericanEuropean Consensus Group revised criteria (Vitali et al., 2002).

\subsection{Peptide synthesis}

Peptides were synthesized as previously described (Kampylafka et al., 2011). Briefly, eleven peptides in the form of peptide dendrimers 
(multiple antigenic peptides, MAP) were synthesized by Bio-synthesis Inc., U.S.A. Three peptides, previously shown to be the main linear target epitopes of AQP4 autoantibodies, were selected for the present study. AQPpep1 (aa 1-22, MSDRPTARRWGKCGPLCTRENI) has been designed to include the first 22 amino acids that are included only in the $\mathrm{M}_{1}$ isoform of the aquaporin 4 protein. AQPpep4 (aa 88-113, FGHISGGHINPAVTVAMVCTRKISIA) corresponds to the first intracellular loop of the molecule while AQPpep8 (aa 252-275, FCPDVEFKRRF KEAFSKAAQQTKG) represents a key epitope in the C-terminus intracellular domain.

\subsection{ELISA-AQP4 assays}

All patients and controls were evaluated for the presence of autoantibodies against the selected peptides by ELISA assays. 96-well microtitre plates were coated with $100 \mu \mathrm{l}$ of peptide solution $(2.5 \mu \mathrm{g} / \mathrm{ml}$ in sodium carbonate-sodium bicarbonate buffer, $\mathrm{pH} 9.8$ ) and kept at $4{ }^{\circ} \mathrm{C}$ overnight. Afterwards, the remaining binding sites were blocked with $2 \%$ bovine serum albumin (BSA) in phosphate buffered saline (PBS) at room temperature for 1 hour. After 3 washes with PBS, sera were added in 1/150 in 2\% BSA/PBS dilution. After a 2-hour incubation and 3 washes with PBS, alkaline phosphate conjugated antihuman $\operatorname{IgG}$, diluted $1 / 1200$ in $2 \%$ BSA/PBS, was added for 1 hour at room temperature and color development was quantified at $405 \mathrm{~nm}$. The cut-off values for each peptide assay were determined using the mean Optical Density plus 3 standard deviations of the sera from the 28 healthy controls.

Homologous inhibition experiments were performed, in order to evaluate the specificity of the binding. These assays were initially performed with variations of serum and peptide concentrations in pretreatment dilutions, in order to define the relative concentrations that would provide optimal results. The rest of the ELISA conditions (i.e. temperature, duration of incubation, diluting and washing buffers) remained the same. In this context, serum dilutions of $1 / 150,1 / 300$ and $1 / 500$ were each combined with peptide concentrations of $0-4-16-64 \mu \mathrm{g} / \mathrm{ml}$ and 0 $10-40 \mu \mathrm{g} / \mathrm{ml}$, in order to define optimal conditions. An inhibition assay was considered to be positive when the inhibition percentages for the examined peptide were $\geq 50 \%$, and the reactivity range between the examined peptide and the control peptide was $\geq 40 \%$. Following assay optimization all positive MS and control sera were tested. Serum was pretreated in $1 / 300$ dilution with increasing concentrations $(0 \mu \mathrm{g} / \mathrm{ml}$, $10 \mu \mathrm{g} / \mathrm{ml}$ and $40 \mu \mathrm{g} / \mathrm{ml}$ ) of reactive peptide and control peptide (Ctrl-Pep) and incubated overnight at $4{ }^{\circ} \mathrm{C}$. Subsequently, all sera dilutions were tested by ELISA for reactivity against the peptides.

\subsection{ELISA-MOG assays}

All MS patients and healthy controls were tested for the presence of anti-MOG serum antibodies using a commercial ELISA SensoLyte ${ }^{\circledR}$ anti-Human MOG IgG kit following the manufacturer's instructions.

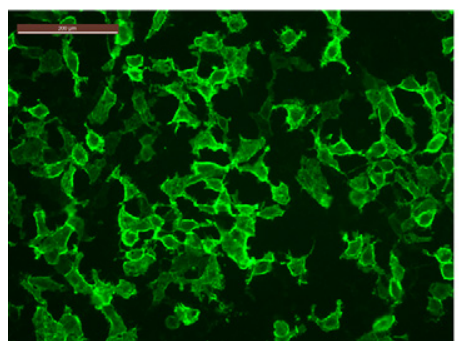

a) NMO patient

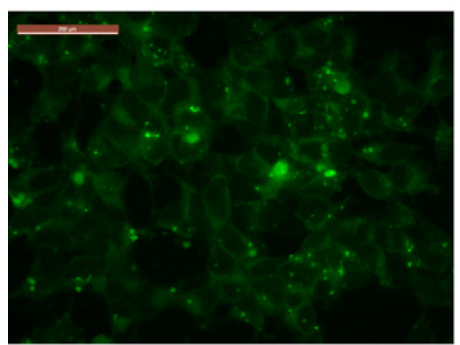

b) RRMS patient

\section{AQPpep8}
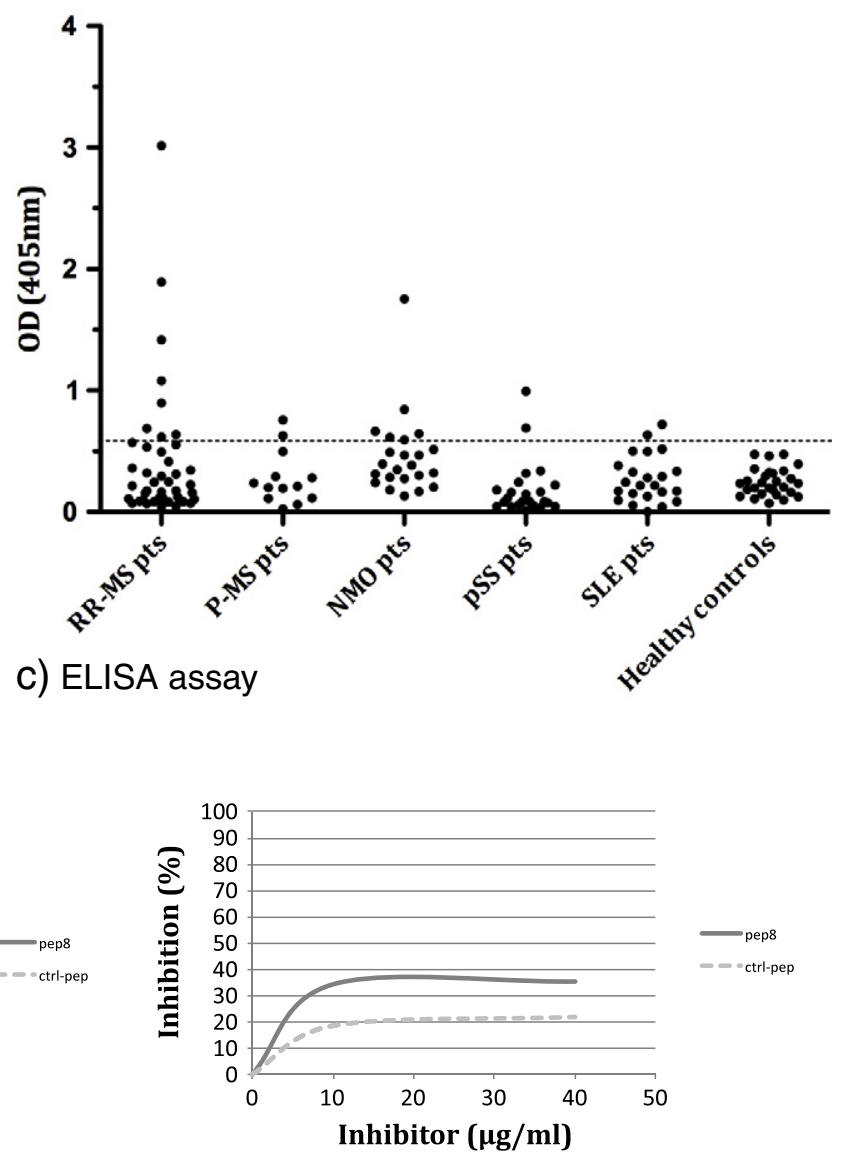

e) MS serum \#19

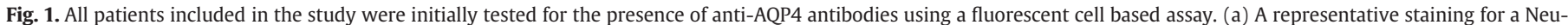

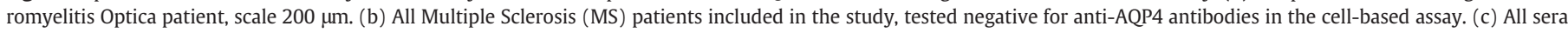

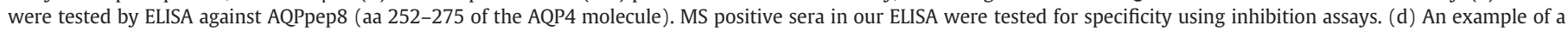
serum exhibiting specific binding. (e) An example of a serum exhibiting non-specific binding. 
Table 1

Patient clinical and laboratory characteristics.

\begin{tabular}{|c|c|c|c|c|c|c|c|c|c|c|c|}
\hline Patient & Sex & $\begin{array}{l}\text { Age at disease } \\
\text { onset }\end{array}$ & $\begin{array}{l}\text { Disease } \\
\text { duration }\end{array}$ & $\begin{array}{l}\text { EDSS } † \text { at } \\
\text { disease onset }\end{array}$ & $\begin{array}{l}\text { EDSS } † \text { at blood } \\
\text { sampling }\end{array}$ & $\begin{array}{l}\text { No of relapses } \\
\text { per year }\end{array}$ & $\begin{array}{l}\text { Optic } \\
\text { neuritis }\end{array}$ & $\begin{array}{l}\text { Spinal } \\
\text { cord }\end{array}$ & $\begin{array}{l}\text { Brain } \\
\text { lesions }\end{array}$ & $\begin{array}{l}\text { IgG } \\
\text { Index }\end{array}$ & OCBs* \\
\hline MS9 & $\mathrm{F}$ & 19 & 3 & 2 & 1 & 5.3 & Yes & Yes & Yes & Positive & Yes \\
\hline MS24 & $\mathrm{F}$ & 30 & 6 & 0 & 1 & 6.9 & Yes & No & Yes & Positive & Yes \\
\hline MS37 & $\mathrm{F}$ & 44 & 5 & 1 & Unknown & 0.5 & Yes & Yes & Yes & Positive & Yes \\
\hline MS39 & $\mathrm{F}$ & 30 & 8 & 1 & 1 & 0.3 & Yes & Yes & No & Positive & Yes \\
\hline MS46 & $\mathrm{F}$ & 26 & 7 & 2 & 3,5 & 3.4 & Yes & No & Yes & Positive & Yes \\
\hline MS60 & $\mathrm{F}$ & 18 & 8 & Unknown & 6 & 2.1 & Yes & Yes & Yes & Positive & Yes \\
\hline
\end{tabular}

\section{Results}

NMO-positive sera, selected by a cell-based assay, exhibited specific reactivity (as attested by the homologous inhibition experiments) against intracellular epitope AQPaa252-275, confirming previous work. As expected, RRMS and PMS sera tested negative for anti-AQP4 antibodies using a fluorescent cell-based assay (Fig. 1b). The same sera however tested by ELISA, showed that 8/45 patients reacted (cut-off $>3 S D$ of the healthy controls) with the peptide AQPaa252-275 (Fig. 1c). Following homologous inhibition experiments, it was shown that the reaction was specific in $6 / 8$ patients (13\% specific reactivity in the RRMS group). (Examples of a positive and a negative inhibition assay are shown in Fig. 1d and e, respectively) No specific reaction was observed in the RRMS group for the other two peptides tested (AQP aa 1-22, AQP aa 88-113). PMS, healthy and disease controls showed no specific reactivity against any of the AQP4 peptides.

All MS patients were also tested for anti-MOG reactivity using ELISA. Our rationale was to test whether our sera were polyreactive, i.e. binding variable antigenic targets. Only one RRMS patient showed positivity higher than 3 standard deviations of the mean of the healthy controls (Supplementary Fig. 1).

\subsection{Clinico-pathological correlations}

All six AQP4-positive RRMS patients were women with a variant age of disease onset (18-44 years old) and disease duration from 3 to 8 years. They were all diagnosed according to the MacDonald criteria, with dispersion in both time and space. All patients had optic neuritis, positive IgG index, oligoclonal CSF bands and MRI findings consistent with MS in the brain and cervical spinal cord. Four patients also had findings in the thoracic spinal cord (Table 1).

\section{Discussion}

We demonstrate that a subset of RRMS patients (13\%) have antibodies against the C-terminal of the AQP4 molecule; none of the patients with RRMS or PMS have antibodies to structural AQP4 epitopes based on a cell-based assay.

A discrepancy was noted between two recent studies; in one, a percentage of classic MS patients harbored anti-AQP4 antibodies based on a cell-based assay, but in a follow-up study in the same ethnic group (Apiwattanakul et al., 2012), no AQP4 positivity was shown, arguing that the NMO spectrum of diseases needs better clinical clarification. Our results support the view that even among Caucasian patients with classic MS, a subset of patients with RRMS exhibits immunological overlap with NMO regarding certain antigenic epitopes on the AQP4 molecule.

To identify whether there were any clinical commonalities between the C-terminal- AQP4 -positive RRMS patients and the NMO-disease spectrum, we retrospectively examined the medical records of the positive RRMS patients. We observed that, a common clinical finding in all six positive patients was that their first clinical manifestation was optic neuritis. Of special interest, five of the six patients had not responded in immunomodulatory treatments and had to switch to immunosuppressive therapy with a good clinical outcome after 3-8 years of follow up and an EDSS score for all 6 patients between 0 and 2.5 .

What is particularly pertinent to NMO, is the observation that the $\mathrm{N}$-terminal domain where the main B-cell epitope resides, is in an area where a significant T-cell epitope is also found (Arellano et al., 2012). In NMO patients, this T-cell epitope resides at amino acids 281-300 adjacent to the most prominent B-cell linear epitope which resides at amino acids 252-275 (Kampylafka et al., 2011). Because both of these epitopes are intracellular and were not found in any other disease tested, the results suggest that in NMO, contrary to the common belief that only the extracellular epitopes are important for disease progression, some extracellular domains may be also relevant, at least in pointing out antigenic similarities between NMO and RRMS patients.

It is still unclear how these prominent intracellular epitopes contribute to NMO pathogenesis and whether the presence of specific antibodies against them plays a role in the pathogenesis of MS. In NMO lesions, complement activation products are found within astrocytes and macrophages, resembling type-II MS lesions (Bruck et al., 2012) Of interest, antibody-independent AQP4 loss, is observed in MS lesions (Matsuoka et al., 2011), suggesting that an astrocytopathy is maybe a feature in some MS patients (Kira, 2011). It is possible, that the affected astrocytes in these MS cases, may release otherwise cryptic epitopes which are then recognized by the immune system, as proposed for several systemic autoimmune diseases (i.e.Lupus) (Routsias et al., 2006). Likewise, it can be hypothesized that in NMO both T- and Bcell intracellular epitopes may be periodically exposed after the initial astrocytic damage, thereby contributing to sudden relapses. As the factors that trigger a relapse in MS patients remain unknown, the proposed hypothesis that AQP4 epitopes are exposed during astrocytic damage might be also applicable to MS. It is notable however, that our observations were specific for the RRMS, as none of the patients with PMS or any of the other autoimmune disease controls tested, such as SLE or Sjogren's syndrome, which often associated with NMO, recognized the C-terminal AQP-4 epitope. Also, the positive RRMS patients did not represent a polyreactivity phenomenon because they did not recognize other relevant antigenic targets such as MOG. Further work is needed to elucidate the pathogenic significance of these antibodies.

Supplementary data to this article can be found online at http:// dx.doi.org/10.1016/j.jneuroim.2013.04.017.

\section{References}

Apiwattanakul, M., Asawavichienjinda, T., Pulkes, T., Tantirittisak, T., Hemachudha, T., Horta, E.S., Jenkins, S.M., Pittock, S.J., 2012. Diagnostic utility of NMO/AQP4-IgG in evaluating CNS inflammatory disease in Thai patients. J. Neurol. Sci. 320, 118-120.

Arellano, B., Hussain, R., Zacharias, T., Yoon, J., David, C., Zein, S., Steinman, L., Forsthuber, T., Greenberg, B.M., Lambracht-Washington, D., Ritchie, A.M., Bennett, J.L., Stuve, O., 2012. Human Aquaporin 4281-300 is the immunodominant linear determinant in the context of HLA-DRB1*03:01: relevance for diagnosing and monitoring patients with Neuromyelitis Optica. Arch. Neurol. 69, 1125-1131.

Bruck, W., Popescu, B., Lucchinetti, C.F., Markovic-Plese, S., Gold, R., Thal, D.R., Metz, I., 2012. Neuromyelitis optica lesions may inform multiple sclerosis heterogeneity debate. Ann. Neurol. 72, 385-394.

Hochberg, M.C., 1997. Updating the American College of Rheumatology revised criteria for the classification of systemic lupus erythematosus. Arthritis Rheum. 40, 1725. 
Kampylafka, E.I., Routsias, J.G., Alexopoulos, H., Dalakas, M.C., Moutsopoulos, H.M., Tzioufas, A.G., 2011. Fine specificity of antibodies against AQP4: epitope mapping reveals intracellular epitopes. J. Autoimmun. 36, 221-227.

Kira, J., 2011. Autoimmunity in neuromyelitis optica and opticospinal multiple sclerosis: astrocytopathy as a common denominator in demyelinating disorders. J. Neurol. Sci. 311, 69-77.

Lennon, V.A., Kryzer, T.J., Pittock, S.J., Verkman, A.S., Hinson, S.R., 2005. IgG marker of optic-spinal multiple sclerosis binds to the aquaporin-4 water channel. J. Exp. Med. 202, 473-477.

Lucchinetti, C., Bruck, W., Parisi, J., Scheithauer, B., Rodriguez, M., Lassmann, H., 2000. Heterogeneity of multiple sclerosis lesions: implications for the pathogenesis of demyelination. Ann. Neurol. 47, 707-717.

Matsuoka, T., Suzuki, S.O., Suenaga, T., Iwaki, T., Kira, J., 2011. Reappraisal of aquaporin4 astrocytopathy in Asian neuromyelitis optica and multiple sclerosis patients. Brain Pathol. 21, 516-532.
Routsias, J.G., Vlachoyiannopoulos, P.G., Tzioufas, A.G., 2006. Autoantibodies to intracellular autoantigens and their B-cell epitopes: molecular probes to study the autoimmune response. Crit. Rev. Clin. Lab. Sci. 43, 203-248.

Siritho, S., Nakashima, I., Takahashi, T., Fujihara, K., Prayoonwiwat, N., 2011. AOP4 antibody-positive Thai cases: clinical features and diagnostic problems. Neurology 77, 827-834.

Srivastava, R., Aslam, M., Kalluri, S.R., Schirmer, L., Buck, D., Tackenberg, B. Rothhammer, V., Chan, A., Gold, R., Berthele, A., Bennett, J.L., Korn, T., Hemmer, B., 2012. Potassium channel KIR4.1 as an immune target in multiple sclerosis. N. Engl. J. Med. 367, 115-123.

Vitali, C., Bombardieri, S., Jonsson, R., Moutsopoulos, H.M., Alexander, E.L., Carsons, S.E. Daniels, T.E., Fox, P.C., Fox, R.I., Kassan, S.S., Pillemer, S.R., Talal, N., Weisman, M.H., European Study Group on Classification Criteria for Sjogren's, S., 2002. Classification criteria for Sjogren's syndrome: a revised version of the European criteria proposed by the American-European Consensus Group. Ann. Rheum. Dis. 61, 554-558. 